\title{
Otras enfermedades periodontales. I: Periodontitis como manifestación de enfermedades sistémicas
}

\author{
SANZ-SÁNCHEZ I* \\ BASCONES-MARTÍNEZ A**
}

\begin{abstract}
Sanz-Sánchez I, Bascones-Martínez A. Otras enfermedades periodontales. I: Periodontitis como manifestación de enfermedades sistémicas. Av Periodon Implantol. 2008; 20, 1: 59-66.
\end{abstract}

\begin{abstract}
RESUIMEN
Introducción: En la última clasificación que se ha realizado de las enfermedades periodontales (World Workshop de 1999), se ha modificado la asignación de periodontitis como manifestación de enfermedades sistémicas. El objetivo de este trabajo es conocer las distintas enfermedades que pueden estar asociadas en mayor o menor grado con alteraciones a nivel del periodonto.

Material, métodos y resultados: Para la realización de este trabajo se han analizado 12 artículos publicados en revistas científicas internacionales y nacionales. Para la búsqueda se han empleado la base de datos MEDLINE y Cochrane.

Discusión: Dentro de las enfermedades sistémicas que pueden afectar al periodonto, nos podemos encontrar con tres grupos: alteraciones hematológicas, alteraciones genéticas y un grupo de enfermedades que no pueden ser especificadas de otra manera.
\end{abstract}

\section{PALABRAS CLAVE}

Periodontitis, systemic disease, Down syndrom, World workshop, Leukemia.

Fecha de recepción: Noviembre 2007.

Aceptado para publicación: Diciembre 2007.

\section{INTRODUCCIÓN}

Las clasificaciones de las enfermedades periodontales han ido cambiando a lo largo de las últimas décadas. Se ha tratado de buscar un consenso que pusiese de acuerdo a las distintas asociaciones mundiales, pero esto no ha sucedido y en su lugar han ido surgiendo nuevas clasificaciones que tratasen de corregir los defectos de las anteriores. En primer lugar cabe destacar la clasificación americana de 1989 ( $1^{\text {er }}$ Worl Workshop), que dividía las enfermedades periodon- tales en: a) Periodontitis del Adulto, b) Periodontitis de comienzo temprano, c) Periodontitis asociadas a enfermedades sistémicas y, d) Enfermedades periodontales necrotizantes. Esta clasificación se basaba en la edad del paciente y en la tasa de progresión de la pérdida ósea, con las limitaciones que ello conlleva, debido a que la edad de diagnóstico de la enfermedad no tiene por qué coincidir con la edad de aparición y para poder establecer una tasa de progresión serían necesarias por lo menos dos visitas. Los europeos trataron de corregir estas carencias clasificando

Alumno Máster en Ciencias Odontológicas y Periodoncia e Implantes. Facultad de Odontología. Universidad Complutense de Madrid.

** Catedrático de Medicina Bucal y Periodoncia. Facultad de Odontología UCM. Director Máster en Periodoncia e Implantes (UCM). 
las enfermedades periodontales según un descriptor primario (Periodontitis del adulto, Periodontitis de Aparición Temprana y Periodontitis Necrotizante) y otro secundario que marca la progresión, características microbiológicas, respuesta al tratamiento, características clínicas, etc (European Workshop 1993). Sin embargo, la asociación americana de periodoncia en el $2^{\circ}$ World Workshop de 1999, desarrollan una última clasificación, en la que se incluyen los siguientes apartados y en la que se incluyen los siguientes cambios:

- Se sustituye el término de periodontitis del adulto por periodontitis crónica.

- Desaparece el término de periodontitis de comienzo temprano y se incorpora el de periodontitis agresivas.

- Se reclasifican las enfermedades periodontales como manifestación de enfermedades sistémicas, en las que se incluye la periodontitis prepuberal.

- Se renombran las enfermedades periodontales necrotizantes.

- Se añaden las enfermedades gingivales, abscesos periodontales, lesiones endo-periodontales y condiciones $y / o$ deformidades del desarrollo o adquiridas $(1,2,3,4)$.

Actualmente podemos decir que ninguna de las clasificaciones es perfecta y que cada una tiene sus defectos. Lo más importante es conocerlas y saber las limitaciones que poseen. El objetivo de este trabajo es conocer la clasificación de periodontitis como manifestaciones de enfermedades sistémicas del World Workshop de 1999.

\section{MATERIAL, MÉTODOS Y RESULTADOS}

Para la realización de esta revisión hemos analizado 12 artículos publicados en revistas científicas con índice de impacto internacional y en otras revistas nacionales y libros de texto.

Para la búsqueda hemos empleado las bases de datos MEDLINE en www.pubmed.com y Cochrane. En una primera búsqueda se encontraron numerosos artículos, para lo que se decidió establecer los siguientes criterios:

- Idioma: inglés

- Artículos de: 1998 a 2007

- Dental journals

- Palabras clave: periodontitis, systemic disease, Down syndrom, World workshop, Leucemia.

\section{DISCUSIÓN}

Dentro de este grupo de enfermedades, Armitage propone tres subgrupos de alteraciones que nos pueden modificar el diagnóstico de nuestros pacientes y con ello el modelo de tratamiento que se les va a realizar.

\section{A. ASOCIADAS A ALTERACIONES HEMATOLÓGICAS}

\section{NEUTROPENIA CUANTITATIVA}

Existe un déficit en la cantidad de neutrófilos circulantes en sangre, con lo que se produce un aumento importante de la susceptibilidad a las infecciones. Entre los tipos de neutropenia adquirida encontramos:

- Inducida por drogas, que a su vez puede ser predecible (citostáticos e inmunosupresores) o idiosincrásica (fenotiacidas, aminopiridina, sulfamidas, etc).

- Postinfecciosa.

- Transplante de médula ósea.

- Irradiación.

- Deficiencias nutricionales: vitamina $B_{12}$, folatos, cobre.

Dentro de las neutropenias congénitas destaca la neutropenia cíclica, la cuál se transmite siguiendo un patrón autosómico dominante (5).

\section{LEUCEMIA CUANTITATIVA}

La leucemia es un trastorno hematológico maligno con proliferación anormal y desarrollo de leucocitos y sus precursores en sangre y médula ósea. Puede comprometer cualquiera de los subgrupos de leucocitos, leucocitos polimorfonucleares, linfocitos (importantísimos en la inmunidad adquirida del huésped) y monocitos. La hematopoyesis normal está suprimida y en la mayoría de los casos de leucemia, las células blancas aparecen en sangre circulante en formas inmaduras. La proliferación celular en la leucemia a expensas de líneas celulares hematopoyéticas causa daño a la médula ósea y disminuye el recuento de las células sanguíneas. Como consecuencia el individuo puede morir a causa de infecciones o hemorragias asociadas con neutropenia y trombocitopenia. La clasificación de la leucemia se basa en el curso que sigue la enfermedad, es decir, aguda o crónica, y en el origen de las células involucradas. Las formas básicas son: leucemia linfocítica aguda (LLA) leucemia mielogénica aguda (LMA), leucemia linfocítica crónica (LLC) y leucemia mielogénica crónica (LMC). 
La más frecuente es la leucemia linfocítica crónica, que suele acontecer a partir de los 40 años (6).

\section{Síntomas generales:}

- Anemia.

— > riesgo de infecciones y hemorragias.

\section{Signos visibles en boca:}

Las manifestaciones orales de la anemia pueden soponer un diagnóstico precoz de la misma, incluso pueden mostrar el estado y gravedad de la enfermedad. Los más frecuentes son:

- Adenopatías regionales.

- Mucosas blanquecinas.

- Tumefacción general de la encía asociada a placa

- Inflamación, sangrado y petequias (trombocitopenia).

- Ulceraciones necrosantes gingivales recurrentes.

— Signos sistémicos.

\section{Terapia:}

Distinguimos dos fases:

- La primera es la de remisión y consolidación.

- La segunda es la de mantenimiento. Se deberán llevar a cabo profilaxis regulares, atraumáticas y valorando siempre la necesidad de una cobertura antibiótica según el recuento sanguíneo de los pacientes. Hay que tener siempre en cuenta que los

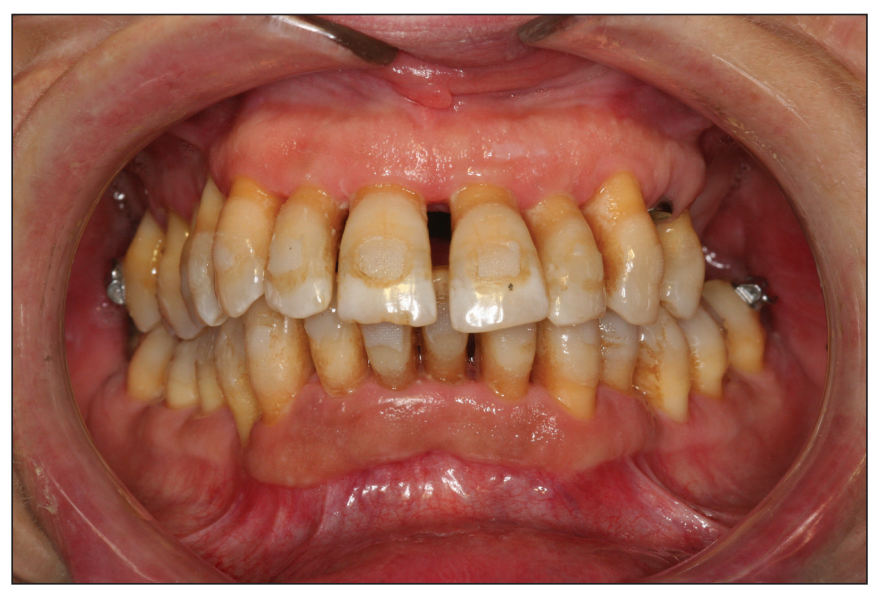

Fig. 1: Visión intraoral de un paciente con leucemia. pacientes con leucemia pueden estar sometidos a quimioterapia $(7,8)$.

\section{Otras (alteraciones Plaquetarias, de cÉlULAS ROJAS...)}

No existe evidencia de que en estas alteraciones aumenten la susceptibilidad de la periodontitis.

\section{B. ASOCIADAS A ALTERACIONES GENÉTICAS}

\section{NEUTROPENIA FAMIILIAR Y CÍCLICA}

La neutropenia cíclica se transmite siguiendo un patrón autosómico dominante. Es una enfermedad que cursa con un déficit de neutrófilos. Se ha identificado en estos pacientes mutaciones en el gen de la elastasa del neutrófilo (ELA2). Se caracteriza por una neutropenia muy severa $\left(<200\right.$ células $\times 10^{6} /$ litro) de 3-6 días de duración, que se repite cada 21 días, aunque en algunos casos los ciclos de hematopoyesis pueden variar entre 2 y 5 semanas. Respecto a las manifestaciones orales los niveles cíclicos de neutrófilos se han asociado ocasionalmente a bolsas periodontales profundas y pérdida ósea extensa y generalizada, úlceras orales y enfermedad periodontal avanzada.

\section{SÍNDROME (SD.) DE DOWN}

El SD es una enfermedad hereditaria resultado de la trisomía del cromosoma 21. Este síndrome es causa retraso mental de distintos grados.

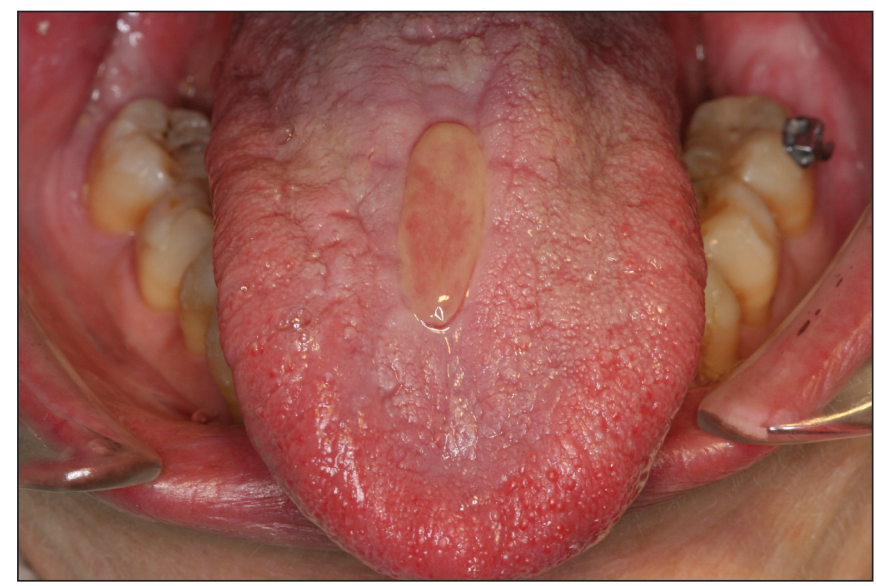

Fig. 2: Visión de una úlcera lingual de un paciente con leucemia. 


\section{Alteraciones asociadas}

Alguna de las características que presentan son: alteraciones cardíacas congénitas (defectos septrales, prolapso de la válvula mitral, defecto septal ventricular o atrio ventricular), desórdenes cardíacos asociados a hipertensión pulmonar, susceptibilidad a padecer endocarditis infecciosa, inmunocompromiso (se manifiesta en frecuentes infecciones, sobre todo respiratorias, como pueden ser TBC, pneumonía, sinusitis, etc), epilepsia, diabetes o hipertiroidismo.

\section{Posibles mecanismos}

Degranulación de metaloproteinasas de la matriz (MMP's), inmunodeficiencia de células T, defectos funcionales en neutrófilos, alteraciones de la síntesis de colágeno.

\section{Clínica:}

- Periodontalmente, inflamación grave en más de la mitad de los pacientes. Mayor destrucción ósea y niveles aumentados de MMP's en fluido crevicular gingival y en saliva.

- Hiper-inervación gingival.

- Aunque presenten problemas de aprendizaje, son sujetos amigables y cooperativos en la consulta. Según las posibilidades de mantener una relación con el paciente, nos plantearemos el uso de premedicación, restricción física, sedación o anestesia general.

- Considerar los fármacos que toma el paciente.

- Se pueden tratar bajo $\mathrm{AL}$, pero muchas veces la sedación es necesaria.

- La AG se hará por un especialista por las posibles complicaciones que pueden surgir asociadas a las patologías que presentan.

- Alteraciones orofaciales: microcefalia, nariz pequeña y baja, hipotonía en mejillas y labios, macroglosia, lengua agrietada, labios hipotónicos, secos y fisurados, frecuentes maloclusión por hipoplasia de maxilares, protrusión mandibular (clase III+ mordida abierta), empuje lingual, paladar estrecho, respiración bucal, sequedad de boca, úvula bífida, labio y paladar fisurados, alteración de erupción de dientes permanentes, agenesias, supernumerarios, retraso en la erupción, microdoncias, hipoplasia e hipocalcificaciones de esmalte.

- Presentan higiene oral deficiente, lo que da caries y enfermedad periodontal frecuentes. Con frecuencia presentan gingivitis ulcerativa (9).

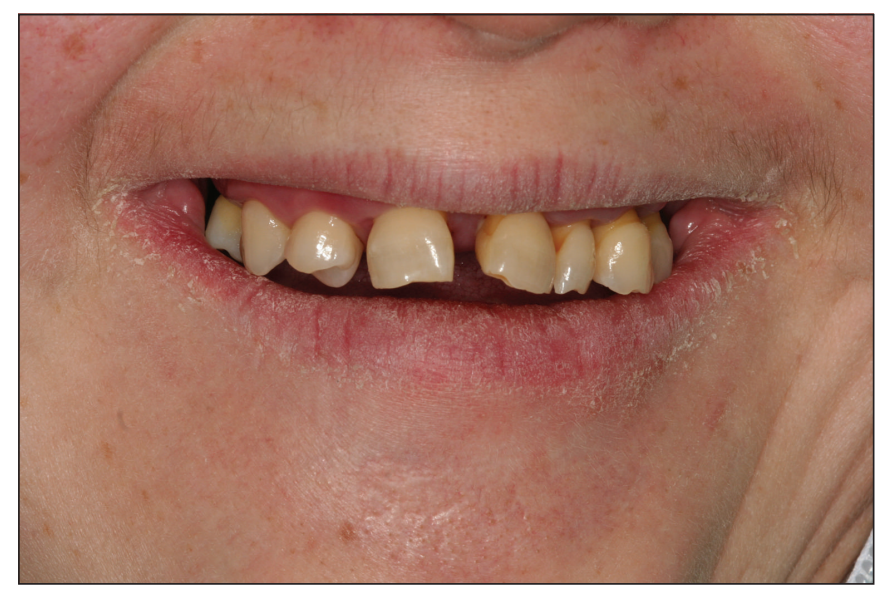

Fig. 3: Visión de la sonrisa de un paciente con síndrome de Down en la que observamos sequedad labial, agenesias o malposicones.

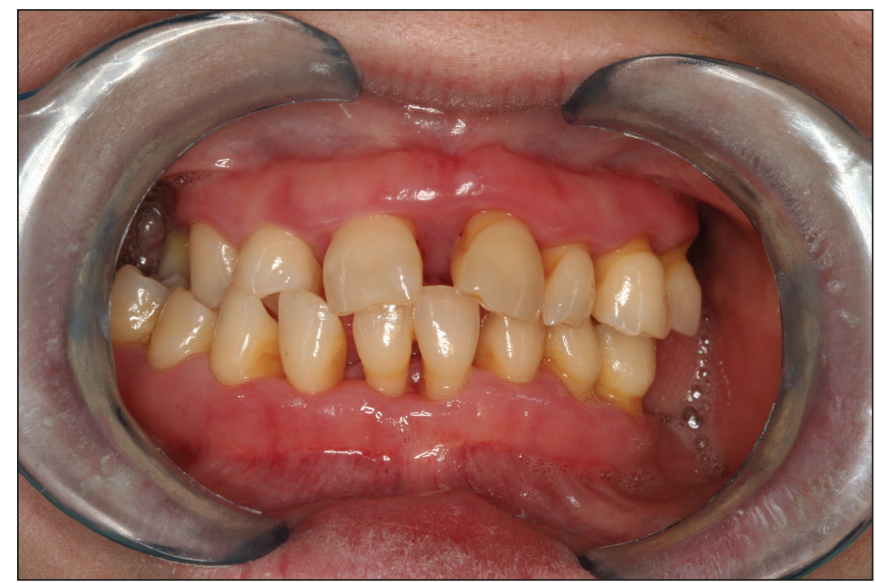

Fig. 4: Visión intraoral de un paciente con síndrome de Down.

\section{SD. DE DEFICIENCIA DE ADHESIÓN LEUCOCITARIA}

Rara enfermedad autosómica recesiva en la cuál, los neutrófilos son incapaces de adherirse a la superficie del endotelio debido a una alteración de las integrinas y/o selectinas. Distinguimos tres grupos genéticos de DAL: en DALl una expresión aberrante de la integrina beta-2 dificulta la emigración desde el flujo sanguíneo; en DAL2 hay una deficiencia en de la "sialyl-Lewis-X" que conlleva un pobre enrollamiento y falta de adhesión al endotelio vascular y una variante de DALl en la que la expresión de la integrina beta-2 es correcta pero la función de adhesión que median estas integrinas está afectada (10). 


\section{Clínica:}

- Mayor susceptibilidad a padecer infecciones en la infancia, especialmente por Staphylococos y Gram negativos.

- Exfoliación prematura de dientes temporales y pérdida de hueso alveolar.

- En casos leves a menudo la enfermedad periodontal es el síntoma principal y el más problemático.

\section{HiperQueratosis PALMO-PLANTAR} (SD. DE PAPILLON-LEFÉVRE)

Raro trastorno congénito (1/4.000.000) que afecta a la piel de las palmas y plantas desde la edad de 2-4 años, dando lugar a una hiperqueratosis en los mismos.

\section{Posibles mecanismos:}

- Alteraciones en la quimiotaxis y en la producción de superóxidos por parte de los neutrófilos.

- > susceptibilidad a las infecciones por:

- Defecto fagocitario.

- Alteración en la barrera epitelial del surco gingival.

- Alteraciones en el cemento.

- Alteraciones en la actividad colagenolítica.

- Alteraciones en ligamento periodontal.

- Aumento en la actividad osteoclástica.

\section{Clínica:}

- Rápida destrucción periodontal entorno a dentición primaria.

- La mutación del gen de la catepsina lleva al enfermo a sufrir un gran riesgo de padecer periodontitis agresivas.

- El tratamiento propuesto es un full mouth desinfection, propuesto por Quirynen en 1995.

\section{Sd. DE ChediaK-Higashi}

Enfermedad autosómica recesiva en la que hay presencia de gránulos lisosomiales gigantes en el interior de granulocitos, monocitos y linfocitos.

Mecanismo: Se caracteriza por un defecto lisosómico que genera anomalías en las células sanguíneas y disfunción neutrófila, tanto en la quimiotaxis como en su capacidad fagocítica.

\section{Clínica:}

- Se asocia frecuentemente a periodontitis avanzadas y severas.
- Aparición de gingivitis grave, movilidad dentaria, supuración y ulceraciones en la lengua y en la mucosa bucal (11).

\section{SD. DE HISTIOCITOSIS}

Antiguamente se conocía como histiocitosis $\mathrm{X}$, aunque actualmente se le ha decidido llamar histiocitosis de células de Langerhans.

\section{Mecanismos}

Hay una proliferación difusa de células que muestran una morfología y son inmunofenotípicamente similares a las células de Langerhans.

\section{Clínica:}

- Úlceras necróticas, marcada pérdida ósea y necrosis de tejidos.

- Rx, esta enfermedad se presenta clásicamente como en "dientes flotantes. Puede aparecer un patrón $\mathrm{Rx}$ en sacabocados: lesiones multiloculares expansivas con rebordes escleróticos.

- El diagnóstico debe comprender investigaciones inmunológicas y hematológicas en estadios tempranos, así como biopsia de tejido de granulación (12).

\section{SD. DE ALMACENAJE DE GLUCÓGENO}

Alteración autosómica recesiva muy rara.

\section{Clínica:}

- Bajo número de neutrófilos, alteración en el metabolismo de hidratos de carbono y enfermedad periodontal.

\section{AGRANULOCITOSIS GENÉTICA INFANTIL}

Patología autosómica recesiva muy rara, cuyo cuadro incluye la ausencia completa de granulocitos periféricos y aumento de monocitos, basófilos y eosinófilos.

\section{Clínica:}

- Frecuentemente enfermedades infecciosas que remiten a lo largo de la vida sin necesidad de intervención.

- Estomatitis generalizada y dolorosa, sangrado espontáneo y tejido necrótico. 
- Frecuentes periodontitis con signos radiográficos de patrón de pérdida ósea en edad temprana con pérdida dentaria (12).

\section{SD. DE COHEN}

Síndrome autosómico recesivo caracterizado por retraso motor y mental no progresivo, obesidad, neutropenia, hipotonía, aracnodactilia, microcefalia, dismorfia craneofacial típica, miopía y distrofia coriorretiniana.

\section{Clínica:}

- Filtrum aumentado, micrognatia, hendidura del paladar, dientes anteriores prominentes, puente nasal alto y orejas malformadas.

\section{SD. DE EHLERS-DaNLOS (TIPOS IV Y VIII)}

Síndromes autosómicos recesivos caracterizados por alteraciones del tejido conectivo donde se producen defectos en la síntesis del colágeno. Está caracterizado por hiperlaxitud articular, hiperextensibilidad de la piel y fragilidad de los tejidos.

Se clasifican en 10 tipos según sus síntomas clínicos.

\section{Clínica:}

- Tipo VIII: Periodontitis generalizada y mucosa frágil que da lugar a múltiples cicatrices. Pérdida prematura de dientes temporales y permanentes.

- La hipoplasia del esmalte se ve comúnmente. Dientes premolares y molares pueden presentar grietas profundas y largas cúspides. Los dientes parecen ser frágiles y la microdoncia a veces se presenta.

- La lengua es muy flexible y aproximadamente el $50 \%$ de personas con el síndrome pueden tocar el extremo de su nariz con su lengua.

- Consideraciones clínicas:

- La presencia de prolapso de la válvula mitral generalmente indica que los antibióticos profilácticos están indicados para procedimientos relevantes.

- Visitas dentales de duración corta.

- La fuerza usada en el tratamiento ortodóntico debería ser más suave de lo normal, dada la fragilidad del ligamento periodontal. Dado que la recaída es frecuente, es necesario un período más largo de retención.

- Idealmente, la cirugía dental y maxilofacial debería evitarse.

- Es imprescindible probar valores de la coagulación de sangre antes de proceder con cirugía (12).

\section{Hipofosfatasia}

Forma autosómica recesiva (a veces dominante) con niveles disminuidos de fosfatasa alcalina en sangre. Hay cuatro patrones fenotípicos diferentes mediante los cuales se expresa esta enfermedad y todos se asocian a mutaciones en el gen de las fosfatasas alcalinas.

\section{Clínica:}

- Pérdida ósea severa con pérdida prematura de dientes temporales, particularmente los anteriores.

- Ausencia de cemento que no permite la adecuada unión diente-ligamento periodontal mediante las fibras de Sharpey y taurodontismo.

\section{OTRAS ALTERACIONES: ENFERMEDAD GRANULOMATOSA CRÓNICA}

Ligado al cromosoma X.

\section{Clínica:}

- Deficiencia en la capacidad fagocitaria de PMN y monocitos.

- Eritema y úlceras en la encía y mucosa oral, pero sin manifestaciones periodontales.

\section{NO ESPECIFICADAS DE OTRA MANERA (NOS):}

1. Diabetes Mellitus.

2. Medicamentos.

3. Hormonas sexuales: embarazo, menopausia (osteoporosis).

4. SIDA.

5. Factores ambientales:

- Tabaco.

- Estrés.

6. Malnutrición.

7. Pénfigos y penfigoides de localización gingival (Fig. 5).

\section{CONSIDERACIONES A TENER EN CUENTA EN LA CLÍNICA}

- El manejo de estos pacientes exige una estrecha colaboración entre el odontólogo y el médico.

- Las manifestaciones orales pueden preceder a otras manifestaciones, por lo que hay que conocer los signos de las infecciones orales recurrentes en recién nacidos y niños pequeños, como son el sangrado gingival persistente, el eritema o la pérdida de hueso. 


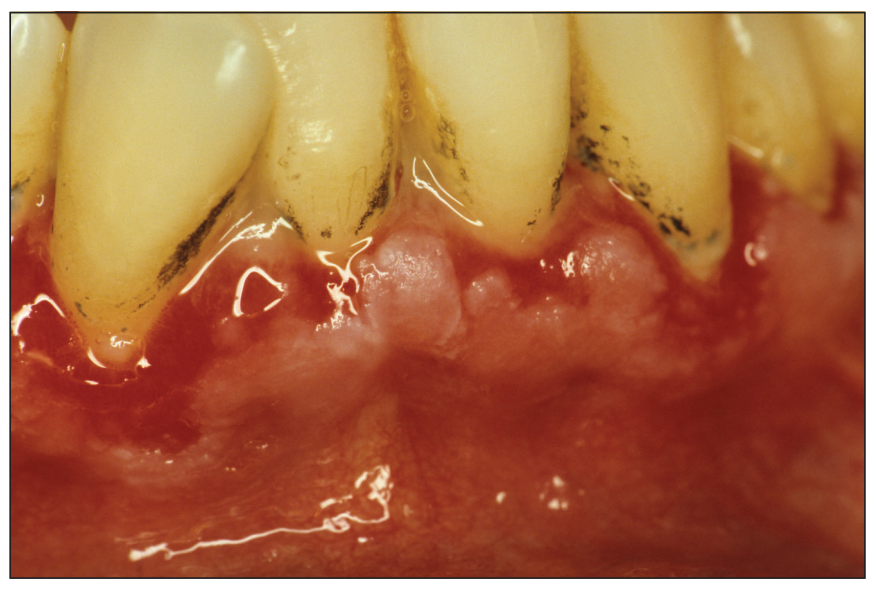

Fig. 5: Pénfigo vulgar de localización gingival.

- Prevención y control de procesos infecciosos intraorales, con el fin de minimizar la necesidad de tratamiento quirúrgico. Por ejemplo, aplicación de flúor tópico, los selladores de fisuras, las recomendaciones dietéticas y la promoción de la salud oral cobran una especial importancia.

- Enjuagues con antisépticos previos a cualquier manipulación odontológica, administración de antibióticos en los días previos y posteriores a la intervención, y el cierre primario del lecho quirúrgico.

- Conocer los efectos de la medicación usada en niños y su impacto en los tejidos orales, y si ese tratamiento podría modificarse para minimizar estos efectos.

- Realizar preventivamente profilaxis periódicas en pacientes sometidos a radioterapia y quimioterapia, ya que en más del $50 \%$ de los casos documentados el riesgo de septicemias es de origen oral.

- Disponemos de factores estimuladores de colonias de granulocitos (G-CSF), de macrófagos (GM-CSF) y la interleuquina -3 (IL-3) como agentes terapéuticos potenciales, cuya utilización permitirá la realización de procedimientos odontológicos como raspajes y alisados, cirugía preprotésica o colocación de implantes dentales.

\section{CONCLUSIONES}

- Las alteraciones sistémicas que afectan a la respuesta del huésped, como inmunodeficiencias primarias, se acompañan muy frecuentemente de periodontitis de aparición temprana.

- La reducción en número o función de PMN conlleva un aumento de riesgo de aparición de periodontitis severa.
- Como se ha visto, la mayoría de las alteraciones sistémicas poseen una base genética por lo que la terapia genética esta siendo ampliamente estudiada para combatir estos defectos.

- La prevención en estos pacientes se convierte en el pilar principal de nuestra labor como odontólogos.

\section{SUMIMARY}

Introduction: In the last classification of periodontal disease (World Workshop, 1999), the assignment of periodontitis as manifestation of systemic disease has been modified. The objective of this paper is to know this systemic disease that can bee associated with different alterations in the periodontum.

Materials, Methods and results: For the preparation of this work, 12 articles published in international and national scientific journals have been analyzed. The MEDLINE and Cochrane databases have been used to make the search.

Disscussion: We can find three different groups of diseases, which can affect the periodontum: hematologyc alterations, genetic alterations and another group that cannot be specified in a different way.

\section{KEY WORDS}

Periodontitis, systemic disease, Down syndrom, World workshop, Leukemia.

\section{BIBLIOGRAFÍA}

1. Armitage G. Development of a classification system for periodontal diseases and conditions. Annals of periodontology/the American Academy of Periodontology. 1999;Dec;4(1):1-6.

2. Brook. O. 1999 International International Workshop for a Classification of Periodontal Diseases and Conditions. Papers. Annals of periodontology/the American Academy of Periodontology. 1999;Dec;4(1:i):1-112.

3. Lindhe Jan KT, Lang Niklaus P. Periodontología clínica e implantología odontológica. $4^{\mathrm{a}}$ edición.Buenos Aires. Editorial: editorial médica panamericana. 2005.

4. Bascones AM. Periodoncia clínica e implantología oral. 2000. 
5. Deas DE, Mackey SA, McDonnell HT. Systemic disease and periodontitis: manifestations of neutrophil dysfunction. Periodontol 2000. 2003;32:82-104.

6. McKenna SJ. Leukemia. Oral Surg Oral Med Oral Pathol Oral Radiol Endod. 2000 Feb;89(2):137-9.

7. HeitzMayfield LJ. Disease progression: identification of high-risk groups and individuals for periodontitis. J Clin Periodontol. 2005;32 Suppl 6:196-209.

8. Quasso L SC, Pavesi L, Calzavara Mantovani D, Perea García MA, Bascones Martínez A. Complicaciones periodontales en las leucemias en edad pediátrica. Avances en Periodoncia. 2005 aug; 17(2).

9. Maita Véliz L CMM. Prevalencia de la enfermedad periodontal en niños con síndrome de Down. Odontol sanmarquina 1998;1(1):31-3.
10. Persson GR. Immune responses and vaccination against periodontal infections. J Clin Periodontol. 2005;32 Suppl 6:39-53.Review.

11. Sollecito TP, Sullivan KE, Pinto A, Stewart J, Korostoff J. Systemic conditions associated with periodontitis in childhood and adolescence. A review of diagnostic possibilities. Med Oral Patol Oral Cir Bucal. 2005 MarApr; 10 (2):142-50.

12. Jordan RC. Diagnosis of periodontal manifestations oof systemic diseases. Periodontology 2000. $2004 ; 34$ : 217-29.

\section{CORRESPONDENCIA}

Ignacio Sanz-Sánchez email: ignaciosanz@mac.com 